Article

\title{
Synchronization in Time-Varying and Evolving Complex Networks
}

\author{
Gualberto Solís-Perales * (D), José Luis Zapata and Guillermo Obregón-Pulido \\ Deptartamento de Electrónica, Centro Universitarios de Ciencias Exactas e Ingenierías, Universidad de \\ Guadalajara, Av. Revolución No. 1500, Col. Olímpica, Guadalajara Jal. C.P. 44430, Mexico; \\ zapata@wizeline.com (J.L.Z.); guillermo.obregon@academicos.udg.mx (G.O.-P.) \\ * Correspondence: gualberto.solis@cucei.udg.mx; Tel.: +52-33-1378-5900 (ext. 27783)
}

Received: 30 September 2020; Accepted: 26 October 2020; Published: 3 November 2020

\begin{abstract}
In this contribution, we present the synchronization in dynamical complex networks with varying couplings. We identify two kinds of variations-(i) Non autonomous (Time-varying) couplings: where the coupling strength depends exclusively on time, (ii) Autonomous or Varying couplings (evolution) where the coupling strength depends on the behavior of the interconnected systems. The coupling strength in (i) is exogenous whereas in (ii) the coupling strength is endogenous and is defined by the states of the systems in the nodes. The exponential stability of the synchronization is ensured for the non autonomous couplings, due to the imposition of the coupling strength. Whereas, in the case of evolutionary couplings the exponential stability of the synchronization is not guaranteed for all time, due to the couplings are not controlled or imposed. We present an overview of these features in complex networks and illustrated by means of numerical examples.
\end{abstract}

Keywords: evolving networks; variable couplings; network synchronization

\section{Introduction}

A complex network is a set of coupled elements with features that do not occur in simple networks such as lattices or random graphs, but often occur when trying to model real-world systems. Many human-made and natural systems are described by models of complex networks, such as the citation network of scientific papers, electrical networks, wireless communication networks, food chains, social networks, ecosystems, and so on [1-6].

The concept of complex networks introduced by Watts and Strogatz (WS) [7] aims to describe the transition from a regular lattice to a random graph. These WS networks exhibit several distinctive traits such as a high degree of clustering and a small average path between any two nodes. Moreover, empirical results by Barabasi and Albert [8] show that large WS networks display scale-free properties, such as the Internet, social networks, and some metabolic networks, to cite some examples. To understand the dynamical behaviors of the complex networks, we may extend the previous models by adding dynamic elements as nodes in the network. One of the most studied phenomena in this kind of network is synchronization, where the networked elements present the same behavior. These dynamical complex networks display various synchronization phenomena between the nodes [9-13]. These synchronization phenomena have attracted increasing attention from various fields of science and engineering.

As an example, Wang and Chen introduced a simple uniform scale-free network model which reflects the complexity of the network structure but still, it refers only to a simple non-varying dynamical network - the coupling strength remains constant for all connections in the network [9]. In the real world, most of the networks we found have different coupling and or varying coupling strengths for distinct nodes. Moreover, many networks we find in nature have varying coupling 
strengths and still exhibit an interesting synchronization phenomenon [14]. For instance, Sorrentino and Ott [15] used an adaptive strategy to guarantee synchronization in a network with non autonomous couplings that evolves under the effects of exogenous (unpredictable) factors. In Reference [16] the authors deals with the problem of synchronization of systems in networks with fix and switching externally defined couplings and communication time delays, for the case of fluctuating structures, it changes or discretely switches the couplings, this is the network couplings changes and remains constant or fixed for a certain time, in our proposal the varying couplings are not constant.

Nevertheless, we observe that many of the existing research about synchronization has been devoted to completely regular networks; this is, networks where the structure and coupling strength remains fixed. Now, we consider possible changes in the links between nodes. In such networks, the changes can be due to the collective behavior (synchronization), or due to the dynamics in the interconnected nodes, or changes in the links between nodes, or a mixture of all of them.

In this note, the synchronization phenomenon in weighted networks is presented, this is, networks where coupling strengths connect the nodes and have different values, moreover, these values are not fixed. The dynamics of these coupling strengths are divided into two; those who depend explicitly on time, and those who depend on the dynamics of the interconnected nodes. Therefore, we claim that the first case is a time-varying network and the latter is an evolving network.

These changes in couplings can be identified as changes in time and changes due to the behavior of the individuals. Changes along time refer to changes in the couplings between nodes which are defined as function purely of time. In this sense, the changes in the network are dictated by an external agent, and the nodes in the networks do not influence their couplings, for instance in Reference [17] the authors consider varying couplings but a control action is implemented to achieve synchronization in a defined reference. Another example is in brain deceases or disorders such as Parkinson, Alzheimer's, and so forth, where external deep brain stimulation is provided to modify the behavior of certain parts of the brain [18-20]. On the other hand, for the evolving couplings, we depart from an analogy with biological systems, where evolution is not only a change along time, but the individuals define it according to their necessity. That is, the couplings in a network can change obeying specific evolving rules defined by the interacting agents. In this sense, the structure of the network is changing along time but defined by the collectivity [21].

Both cases imply possible changes in the network structure. However, the nature of those changes is an important issue. In the first case (non autonomous couplings) the dynamics of the nodes do not determine or define the coupling values and can be considered as an exogenous imposition in the network structure. On the other hand, when the couplings are defined or influenced by the dynamics of the connected nodes, the changes in the network structure are defined collectively, and it is then considered as "evolution" since the behavior of the node produces the network changes. An important issue behind of these cases is the synchronization, on the one hand, the exponential stability of the synchronization is reached since the couplings are imposed and in such a case the stability is controlled. On the other hand, in the case of evolutionary couplings the stability is not controlled and depends absolutely on the coupling behavior and it cannot be controlled.

For dynamical non autonomous couplings in the network, some important assumptions are considered, the functions that describe the coupling strength are chaotic. We also assume that these chaotic functions are positive definite since changes from positive to negative would disconnect the node for an unknown period (when the coupling is 0 ), and that would completely change the network size.

For the case of networks with evolutionary couplings, a function of the nodes states determines the coupling. It is assumed that the function that describes the coupling is the same for any set of two connected nodes, and this function is positive definite.

Research on specific networks that fit this case has been of great interest [22]. Works regarding analysis techniques for human epileptic brain networks [23] and the study of the behavior of dynamical 
networks that evolve slowly as a function of their system properties [24] are examples of the interest in this phenomena.

Our contribution addresses the issue from a more general approach, trying to characterize the generality of these evolving networks and their ability to achieve synchronization.

The paper is organized as follows, in the next section the model for a dynamical network is presented, in Section 3, models for networks with chaotic couplings and evolving couplings are presented, alongside numerical examples to corroborate our results, and in Section 4 some conclusions are provided.

\section{Synchronization of Dynamical Networks}

Consider a typical dynamical network consisting of $\mathrm{N}$ identical linear and diffusively coupled nodes, with each node being an n-dimensional dynamical system. The state equations for the $i$-th node in the network is described by

$$
\dot{x}_{i}=f\left(x_{i}\right)+c \sum_{j=1}^{N} a_{i, j} \Gamma x_{j} \quad i=1,2, \ldots, N .
$$

In this model $x_{i}=\left(x_{i, 1}, x_{i, 2}, \ldots, x_{i, n}\right)^{T} \in \Re^{n}$ are the state variables of node $i, c>0$ represents the coupling strength, $f\left(x_{i}\right) \in \Re^{n}$ is a continuous vector field which describes the dynamical behavior of the node and $\Gamma \in \Re^{n \times n}$ is the outer coupling matrix linking coupled variables. The coupling matrix $A$ is composed by $a_{i, j}$, such that, if there is a connection between node $i$ and node $j(i \neq j)$ then $a_{i, j}=a_{j, i}=1$; otherwise $a_{i, j}=a_{j, i}=0$ and $a_{i, i}=-\sum_{\substack{j=1 \\ j \neq i}}^{N} a_{i, j}$. Suppose that matrix A satisfies

$$
L=\left\{\begin{array}{cc}
a_{i, j} & i \neq j \\
-\sum_{\substack{j=1 \\
j \neq i}}^{N} a_{i, j} & i=j \\
\cdot &
\end{array}\right.
$$

$L$ is the Laplacian matrix of the network that satisfies the dissipative coupling condition given by $\sum_{j=1}^{N} a_{i, j}=0$, therefore, $\lambda_{1}=0$ is an eigenvalue of $L$ associated to the eigenvector $(1,1, \ldots, 1)^{T}$ the eigenvalues of matrix $L$ have all real part less than or equal to $0\left(\lambda_{i} \leq 0\right)$ and if the Laplacian $L$ is irreducible, then the eigenvalue $\lambda_{1}=0$ has multiplicity 1 .

It is said that the nodes in a network synchronize if $x_{1}(t)=x_{2}(t)=\ldots=x_{N}(t)$ and since by the dissipative condition of the Laplacian matrix, the synchronization is achieved in an isolated node $\dot{s}(t)=f(s(t))$ and the solution $s(t)$ can be an equilibrium point, a periodic orbit or a chaotic attractor. Therefore, the stability of the network synchronization depends on the isolated node dynamics, the coupling matrix given by L, the coupling strength and the inner coupling matrix. The following Theorem is a well known result which provides conditions for the synchronization.

Theorem $1([9,10])$. Let us consider the network Equation (1) and let the eigenvalues of $L$ are

$$
0=\lambda_{1}>\lambda_{2} \geq \lambda_{3} \geq \ldots \geq \lambda_{N}
$$

Now suppose that there exists an $n \times n$ diagonal matrix $G>0$ and there are two constants $\bar{d}<0$ and $\sigma>0$ such that

$$
[D f(s(t))+d \Gamma]^{T} G+G[D f(s(t))+d \Gamma] \leq-\sigma I_{n} .
$$

For all $d \leq \bar{d}<0$, with $D f(s(t))$ the Jacobean matrix at the isolated solution $I_{n} \in \Re^{n \times n}$ and moreover if

$$
c \lambda_{2} \leq \bar{d}<0
$$


then the synchronization state is exponentially stable.

Mainly these are the conditions from where the synchronization of complex networks departs $[9,10,25]$. Note that the Laplacian matrix has constant elements, this implies that the connection between nodes does not changes along time which means that there is not any possibility of changing the connection and the strength between nodes. At this point, two possible changes in the connection and the strength are identified. On the one hand, a non autonomous coupling, which only depends on time; this idea in the network represents an arbitrary change where the node dynamic does not interfere, this is, the node dynamics in the network does not determine the network structure, in this case, it is called non autonomous (time-varying) coupling. On the second hand, the couplings depend on the dynamics of the nodes, for this case, the network structure is defined by the node dynamics along the time, therefore it can be seen as an evolution of the network structure, since the nodes define their coupling according to the network requirements, in such a case it is called evolutionary couplings.

\section{Synchronization of Non Autonomous (Time-Varying) and Autonomous Evolutionary Couplings Network}

In this section, the two scenarios previously described are presented. The first one considers a connection that varies chaotically, this is, a linking between nodes which is non autonomous, however, this variation is dictated by an external subsystem, recall that in such a case the node dynamics in the network do not define the connection. In the second scenario, the connections are considered as functions of the states of the connected nodes, that is, an evolutionary coupling, in this case, the nodes define the strength between nodes and have the possibility of defines the network structure.

To represent the two couplings, from (1) $c$ and $a_{i, j}$ in the coupling matrix $A$ are given by $c_{i, j}(x, z, t)$, such that the connection between any nodes vary along time as a function of an external subsystem or evolve according to the node dynamics. Actually, the couplings can be a function or depend on both, an external subsystem and as a function of the nodes in the network, therefore the model of the network is given by

$$
\begin{aligned}
\dot{x}_{i} & =f\left(x_{i}\right)+\sum_{j=1}^{N} c_{i, j}(x, z, t) \Gamma x_{j} \quad i=1,2, \ldots, N . \\
\dot{z} & =g(z),
\end{aligned}
$$

where $x_{i} \in \Re^{n}$ are the state variables for the node $i ; g(z) \in \Re^{m}$ is the vector field of the external subsystem. Let $C(x, z, t)=\left(c_{i, j}(x, z, t)\right)_{N \times N}$ be the matrix couplings in which $c_{i, j}(x, z, t)$ is defined as follows: if there is a connection between nodes $i$ and $j$ at time $t$, then $c_{i, j}(x, z, t)>0$; otherwise $c_{i, j}(x, z, t)=0$ for $j \neq i$. The diagonal elements in $C(x, z, t)$ are defined similar to the diagonal elements on matrix $L$ in (1) given by

$$
c_{i, i}(x, z, t)=-\sum_{\substack{j=1 \\ j \neq i}}^{N} c_{i, j}(x, z, t) \quad i=1,2, \ldots, N .
$$

Let us consider that functions in matrix $C(x, z, t)$ are continuous, bounded, and positive definite since a zero-crossing change the network size (network growth or shrinking). Now the main objective is to achieve synchronization of the nodes in the presence of non autonomous or evolving couplings. Thus, from the model (5) and following Theorem 1, we propose the following

Proposition 1. The synchronization manifold of the network (5) with varying/evolutionary couplings is exponentially stable if there exists a constant value $\bar{d}<0$ such that $\lambda_{2, \max } \leq \bar{d}<0$. 
Proof. Let us first consider that $c=1$ in (4), this means that the coupling strength is now in $c_{i, j}$. Then now let us assume that there exists a constant value $\bar{d}<0$ such that $\lambda_{2, \max } \leq \bar{d}<0$, from the properties of the Laplacian matrix $C(x, z, t)$, which is dissipative for all $t$, it has eigenvalues $\lambda_{1}(x, z, t)=0$ and $\operatorname{Re}\left(\lambda_{p}(x, z, t)\right)<0$ for $p=2, . ., N$ and $0=\lambda_{1}(x, z, t)>\lambda_{2}(x, z, t) \geq \lambda_{3}(x, z, t) \geq$ $\ldots \geq \lambda_{N}(x, z, t)$ thus, without loss generality $\lambda_{2, \min } \leq \operatorname{Re}\left(\lambda_{2}(x, z, t)\right) \leq \lambda_{2, \max }$ for all $t$ and from Theorem 1 , we can consider $\bar{d}=\lambda_{2, \max }$ which satisfies (3) and then the synchronization manifold is exponentially stable.

Note that the value $\lambda_{2, \max }$ is negative for all $t \geq 0$ and this value depends on the weight of the connection between nodes, this can be seen as if there is a particular coupling strength between two nodes and it has a particular weight, then the synchronization relay on the weight of the coupling and it moves the values of the bounds of $\operatorname{Re}\left(\lambda_{p}(x, z, t)\right)$.

\subsection{Network Synchronization with Time Varying Couplings}

We seek complete synchronization in the nodes of a network with non-linear nodes with chaotic couplings where the synchronization manifold is a result of the collective behavior and is not known a priory. We consider a simple scale-free like network with $N=6$. The considered systems for the nodes are the following Lorenz systems given by

$$
\begin{aligned}
& \dot{x}_{1}=\sigma\left(x_{2}-x_{1}\right), \\
& \dot{x}_{2}=x_{1}\left(\rho-x_{3}\right)-x_{2}, \\
& \dot{x}_{3}=x_{1} x_{2}-\beta x_{3},
\end{aligned}
$$

where $\rho=28, \sigma=10$ and $\beta=8 / 3$, and $i$ indicates the node.

The topology of the network illustrated in Figure 1 is given by the coupling matrix $C(t, x)$ and is defined as

$$
C(t, x)=\left[\begin{array}{cccccc}
c_{1,1}(t, x) & 0 & c_{1,3}(t, x) & 0 & 0 & 0 \\
0 & c_{2,2}(t, x) & c_{2,3}(t, x) & 0 & 0 & 0 \\
c_{1,3}(t, x) & c_{2,3}(t, x) & c_{3,3}(t, x) & c_{3,4}(t, x) & 0 & 0 \\
0 & 0 & c_{3,4}(t, x) & c_{4,4}(t, x) & c_{4,5}(t, x) & c_{4,6}(t, x) \\
0 & 0 & 0 & c_{4,5}(t, x) & c_{5,5}(t, x) & 0 \\
0 & 0 & 0 & c_{4,6}(t, x) & 0 & c_{6,6}(t, x)
\end{array}\right]
$$

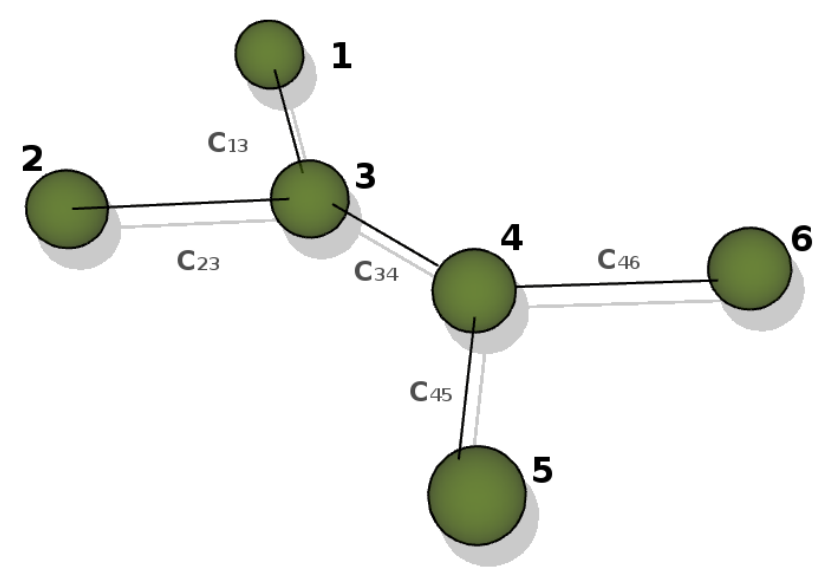

Figure 1. Network topology with varying couplings ci,j. 
To model the variation of the couplings a chaotic unpredictable behavior is considered. The main idea is to induce couplings that are not defined by the connected nodes, this is, couplings that are dictated by an external system or imposed externally. To this end, the Rössler chaotic system is proposed as an external coupling provider since the Rössler system possesses a well-known chaotic behavior. The diagonal elements of the coupling matrix (8) are defined as in (6), functions that comprise the rest of the matrix are given by external functions of the generalized Rössler system given by:

$$
\begin{aligned}
\dot{z}(t) & =\Theta z(t)-z_{M}(t) \boldsymbol{e}_{M-1} \\
\dot{z}_{M}(t) & =\epsilon+b z_{M}(t)\left(z_{m-1}(t)-p\right) .
\end{aligned}
$$

where

$$
\begin{gathered}
\Theta=\left[\begin{array}{ccccc}
a & -1 & 0 & \cdots & 0 \\
1 & 0 & -1 & \ddots & \vdots \\
0 & 1 & \ddots & -1 & 0 \\
\vdots & \ddots & 1 & 0 & -1 \\
0 & \cdots & 0 & 1 & 0
\end{array}\right] \\
z(t)=\left[\begin{array}{c}
z_{1}(t) \\
\vdots \\
z_{M-1}(t)
\end{array}\right] \\
\boldsymbol{e}_{M-1}=\left[\begin{array}{c}
0 \\
\vdots \\
0 \\
1
\end{array}\right] \\
z(t), \boldsymbol{e}_{M-1} \in \Re^{M-1}
\end{gathered}
$$

with $M=5$, one for each coupling, and the parameters $a=0.1, \epsilon=0.1, b=4$, and $p=2$. In order to have positive chaotic functions, each solution of the Rössler system is modified by adding a positive offset, this is, it is known that if the couplings alternates its sign along time, there is a disconnection for a short period of time and more over the behavior of the network could be unstable, therefore a modification of these coupling functions is needed, therefore, the couplings are proposed as $C_{i, j}=z_{i}+\delta_{i}$, with $i, j=1,2, \ldots, 6, C_{i, j}=C_{j, i}$ and $i \neq j, \delta_{i}=25$ is chosen in such a way that the couplings are positive for all time and as a control parameter in the stability of the synchronization error (which will discuss later). Thus in this way there are five different signals as shown in Figure 2. The projections of the chaotic attractor which define the couplings are illustrated in Figure 3. In Figure 4 the error of the state $x_{i, 1}$ are illustrated, where the synchronization error is calculated using the arithmetic average as follows

$$
e_{i}=\left\|M_{i}-x_{i}\right\|
$$

where $M_{i}$ is the mean value of the states. 

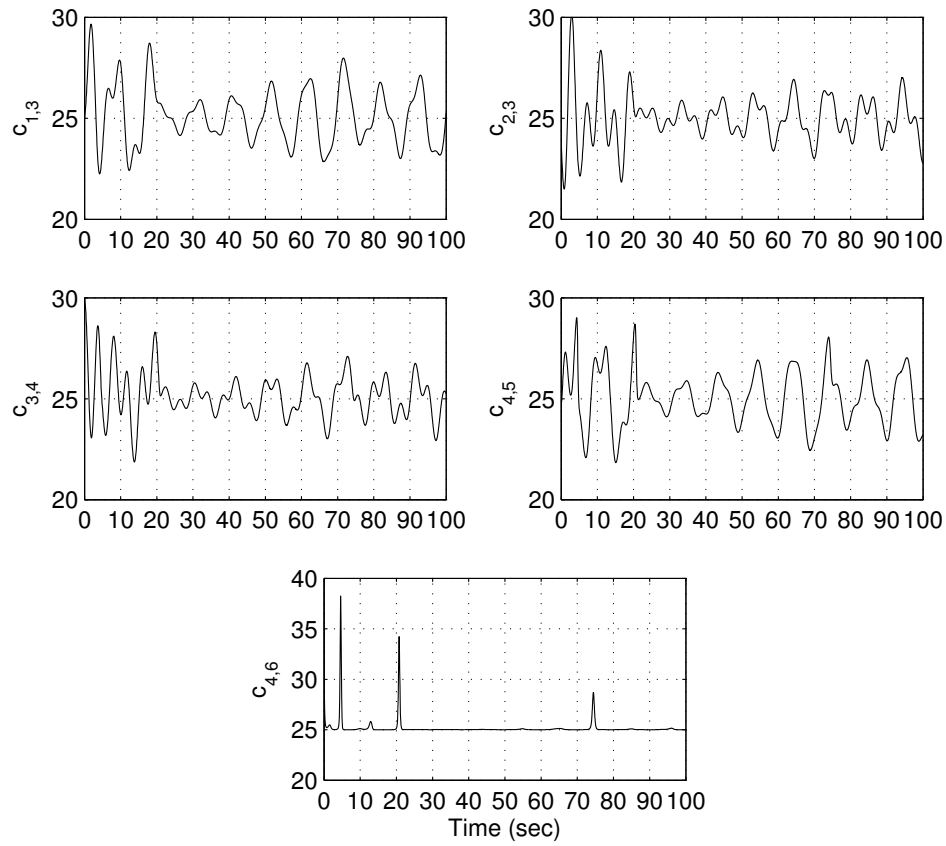

Figure 2. Couplings time-variation obtained from the solutions of the Generalized Rössler System in $\Re^{5}$, with an added offset.
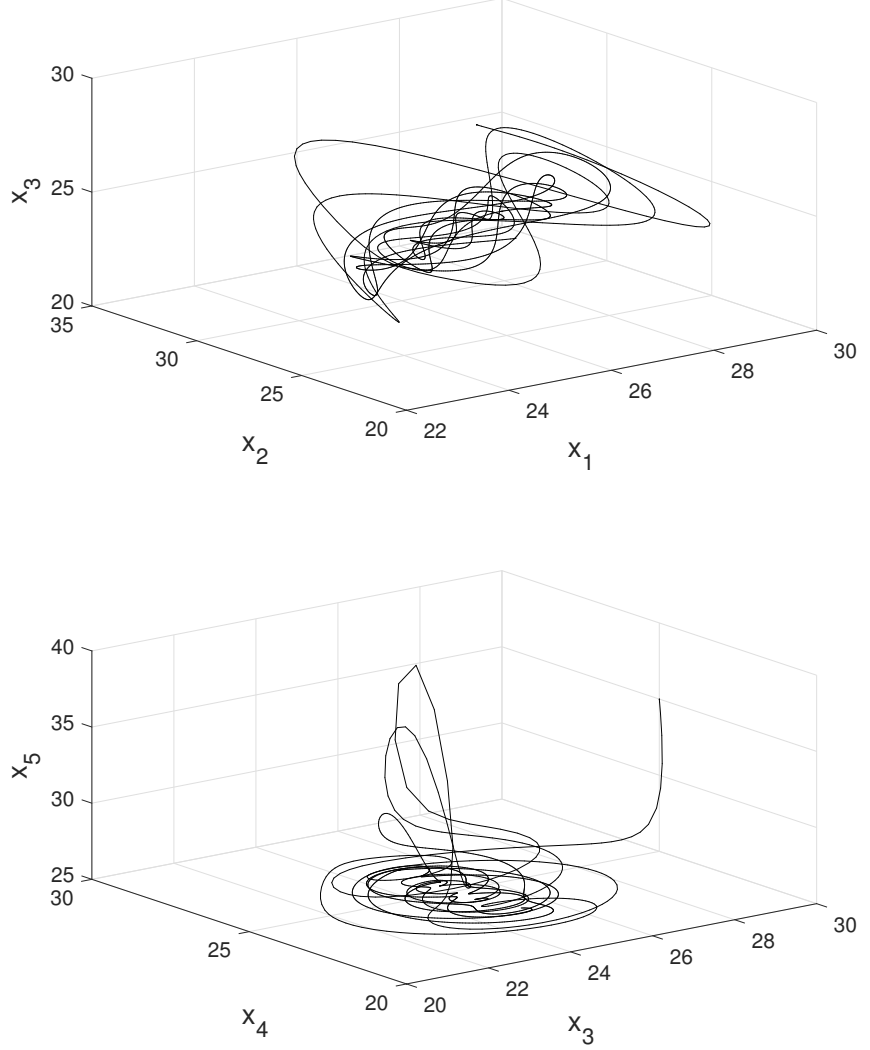

Figure 3. Projections in $\Re^{3}$ of the chaotic attractor in $\Re^{5}$ of the Rössler system which define the couplings between the nodes in the network, this projections include the offset. 

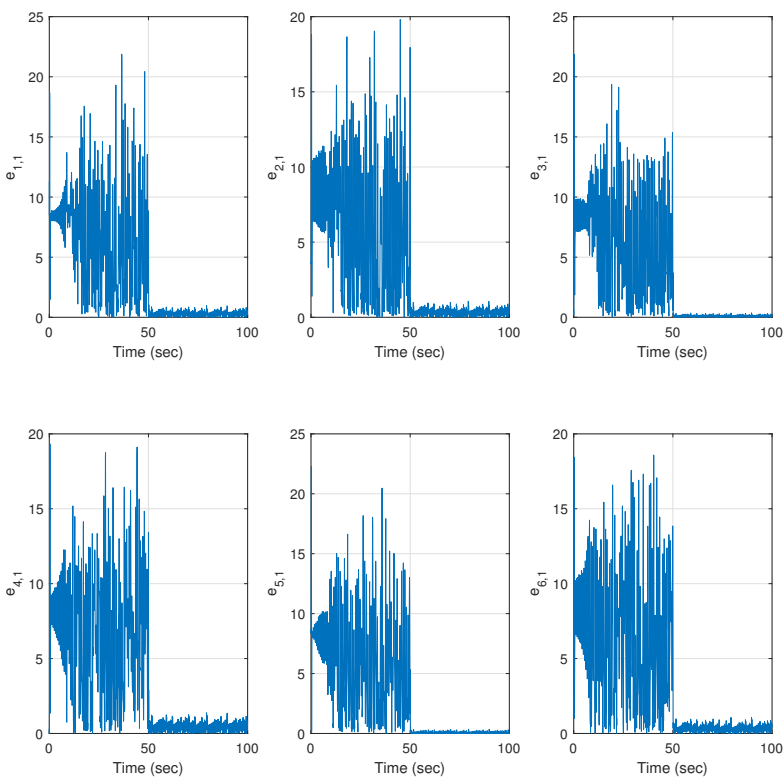

Figure 4. Time evolution of the norm of the synchronization errors between the mean value and the state $x_{i, 1}$.

The behavior of the systems is presented in Figure 5, where the synchronization is achieved even if the couplings are chaotic. In this example, the couplings are non autonomous and unpredictable, thus for some periods the connection between nodes is strong whereas the same coupling debilitates a time after. It is easy to propose any non autonomous couplings, perhaps the minimum value is positive, in some cases could be zero but the nodes lose the connection. The offset is given to satisfy that the eigenvalues of the connection matrix are one zero and the rest of the same sign. In this case, the error between states in the nodes does not define the couplings. This is, the couplings are independent of the behavior in the nodes. For these external non autonomous couplings the behavior of the $\lambda_{2}(t)$ is depicted in Figure 6.
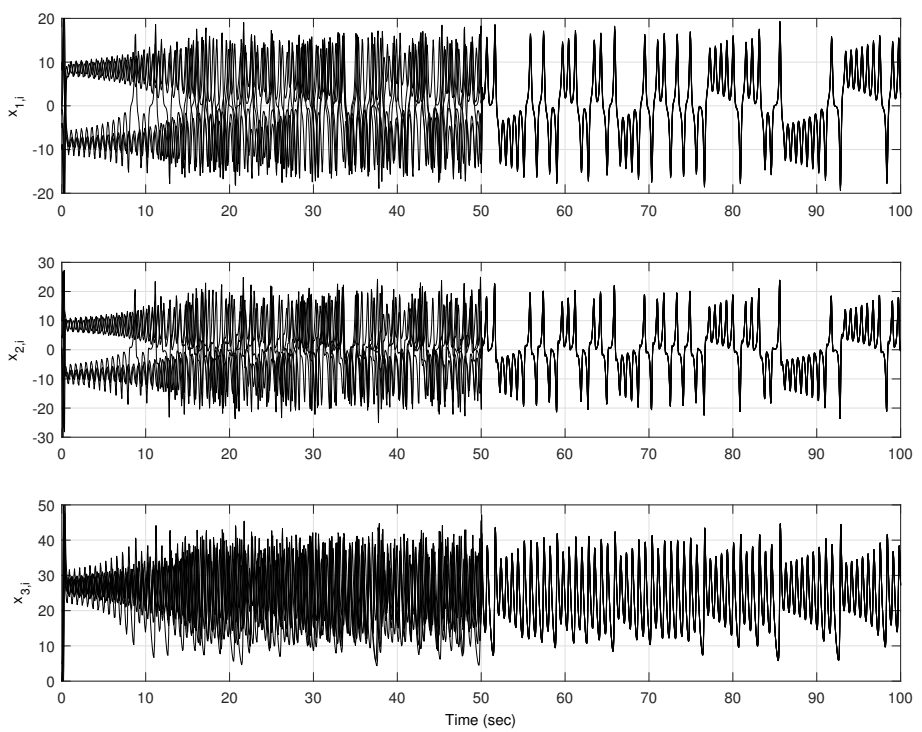

Figure 5. Time evolution of the systems in the nodes connected using chaotic couplings at $t=50 \mathrm{~s}$. 


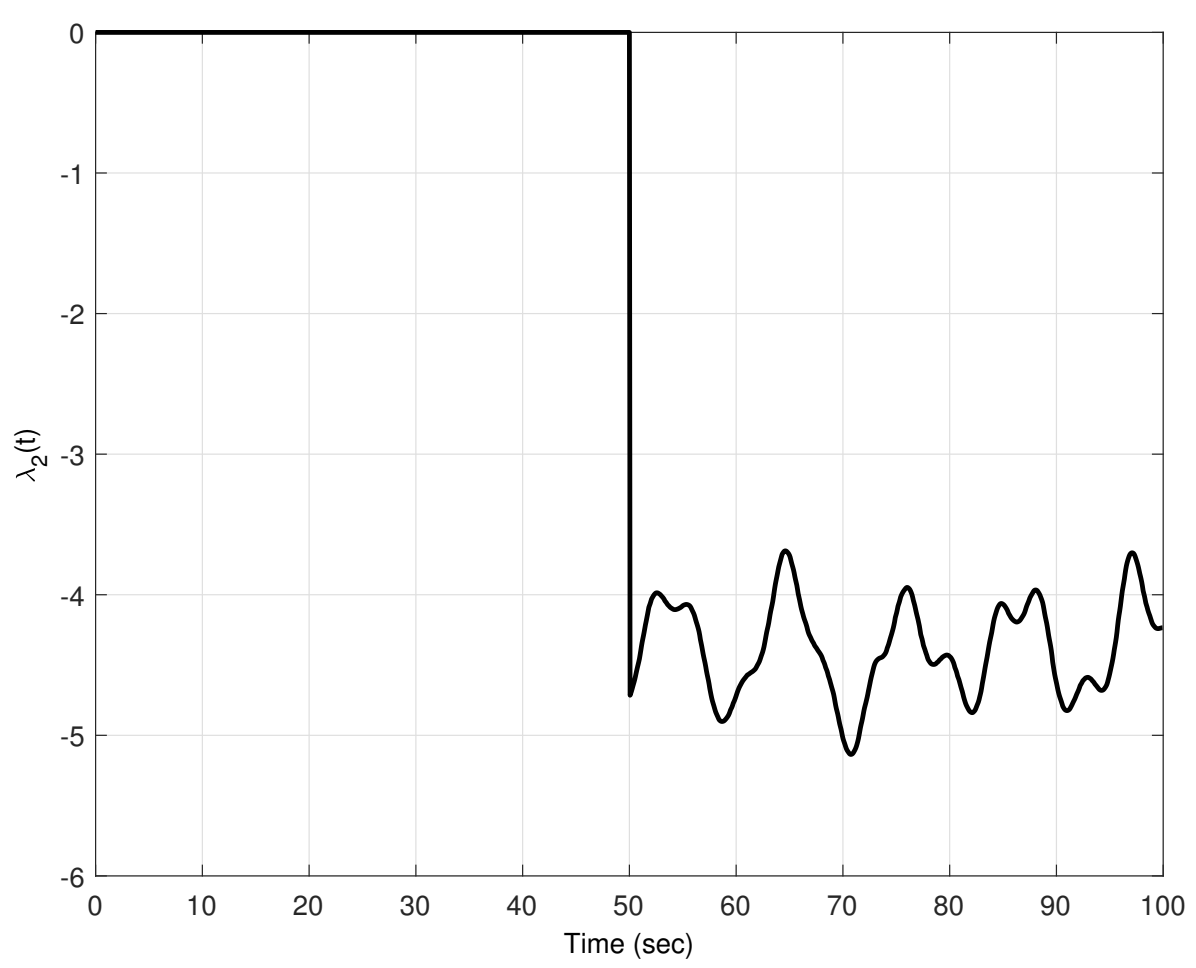

Figure 6. Time evolution of the second largest eigenvalue $\lambda_{2}(t)$ using chaotic couplings.

\subsection{Network Synchronization with Evolutionary Coupling}

As previously stated, one of the biggest problems when trying to model real-world networks is that most of them have inherent structural dynamics. These dynamics are usually modeled as an external system or algorithm that sets coupling values for the network. That is-in most cases-an oversimplification of the problem. In this case, we propose a model with evolving couplings: couplings that vary accordingly to the states of the nodes they connect (which may be seen as their environment).

Note that this definition of the coupling is now a function of the connected systems, whereas, in the case of the non autonomous couplings, they were functions that do not depend on the network's systems behavior. In this sense, the network equation is given by

$$
\dot{x}_{i}=f\left(x_{i}\right)+\sum_{j=1}^{N} c_{i, j}\left(x_{i}, x_{j}\right) \Gamma x_{j} \quad i=1,2, \ldots, N,
$$

where $x_{i} \in \Re^{n}$ are the state variables for the node $i . c_{i, j}\left(x_{i}, x_{j}\right)$ is the evolving coupling between node $i$ and $j$. The evolving couplings are defined as follows: if there is connection between nodes $i$ and $j$ at time $t$, then $c_{i, j}\left(x_{i}, x_{j}\right)>0$, otherwise $c_{i, j}\left(x_{i}, x_{j}\right)=0$ for $j \neq i$. The diagonal elements in the matrix $C\left(x_{i}, x_{j}\right)$ are defined as follows

$$
c_{i, i}\left(x_{i}, x_{j}\right)=-\sum_{\substack{j=1 \\ j \neq i}}^{N} c_{i, j}\left(x_{i}, x_{j}\right) \quad i=1,2, \ldots, N .
$$

As in the case of the varying couplings, for this case the following assumptions are considered the functions that comprise $C\left(x_{i}, x_{j}\right)$ must be continuous, bounded and positive definite. Consider that the coupling function between node $i$ and $j$ is given by $C\left(x_{i}, x_{j}\right)=\sqrt{\left(x_{i}-x_{j}\right)^{2}}$. 
We present the complete synchronization in the nodes of a network of Lorenz systems with evolving couplings where the synchronization manifold is a result of the collective behavior and is not known a priory.

The topology of the network is the same as in Figure 1 and the coupling matrix is given by $C\left(x_{i}, x_{j}\right)$ defined as follows

$$
C\left(x_{i}, x_{j}\right)=\left[\begin{array}{cccccc}
c_{1,1}\left(x_{i}, x_{j}\right) & 0 & c_{1,3}\left(x_{i}, j\right) & 0 & 0 & 0 \\
0 & c_{2,2}\left(x_{i}, x_{j}\right) & c_{2,3}\left(x_{i}, x_{j}\right) & 0 & 0 & 0 \\
c_{1,3}\left(x_{i}, x_{j}\right) & c_{2,3}\left(x_{i}, x_{j}\right) & c_{3,3}\left(x_{i}, x_{j}\right) & c_{3,4}\left(x_{i}, x_{j}\right) & 0 & 0 \\
0 & 0 & c_{3,4}\left(x_{i}, x_{j}\right) & c_{4,4}\left(x_{i}, x_{j}\right) & c_{4,5}\left(x_{i}, x_{j}\right) & c_{4,6}\left(x_{i}, x_{j}\right) \\
0 & 0 & 0 & c_{4,5}\left(x_{i}, x_{j}\right) & c_{5,5}\left(x_{i}, x_{j}\right) & 0 \\
0 & 0 & 0 & c_{4,6}\left(x_{i}, x_{j}\right) & 0 & c_{6,6}\left(x_{i}, x_{j}\right)
\end{array}\right]
$$

where the diagonal elements are defined by (12), and the functions that comprise the rest of the matrix are given by a function of the states on the nodes. Figure 7 illustrates the couplings time evolution, note that this couplings are close to zero when the behavior between nodes are closed each other, but in such a case it seems that the nodes are weakly connected and synchronization could be lost, when synchronization is weak the coupling increases and the synchronization is recovered. Also note that in Figure 7c), the magnitude of the coupling is greater than those of Figure $7 a, b, d)$ this is since $c_{3,4}(x)$ is the coupling that connects nodes 1,2 and 3 to 4,5 and 6 and requires a little more coupling force.
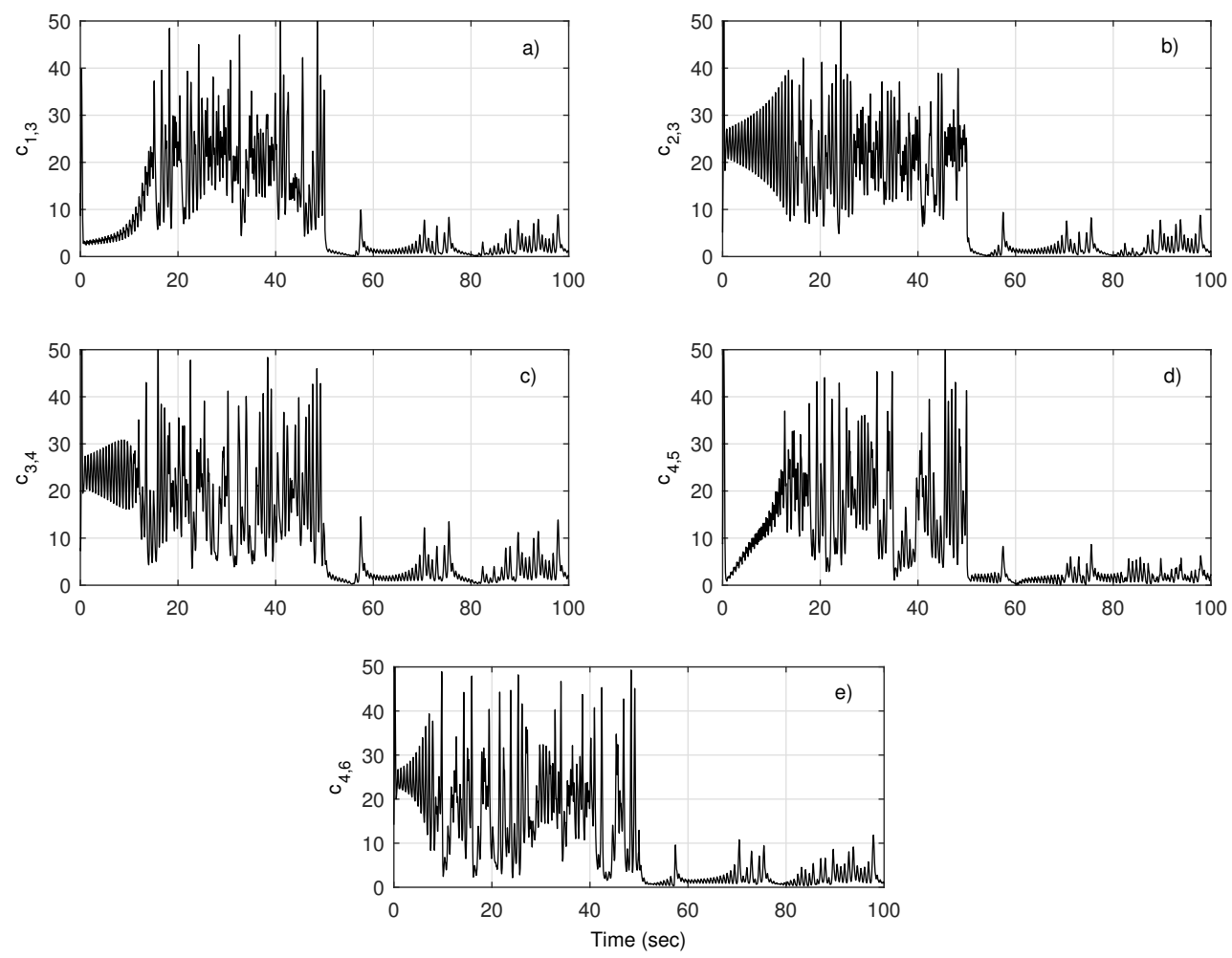

Figure 7. Time evolution of the endogenous couplings between the corresponding nodes. (a) Coupling force between node 1 and node 3, (b) Coupling force between node 2 and node 3, (c) Coupling force between node 3 with node 4 the central nodes and the more strong coupling, (d) Coupling force between node 4 and node 5, (e) Coupling force between node 4 and node 6 . 
Now, with this configuration of the network the systems were initiated without connecting them, this is there is a free behavior until $t=50 \mathrm{~s}$. Figure 8 illustrates the synchronization of the network of Lorenz systems, as it can be seen the synchronization is sustained despite the value of the evolutionary couplings.
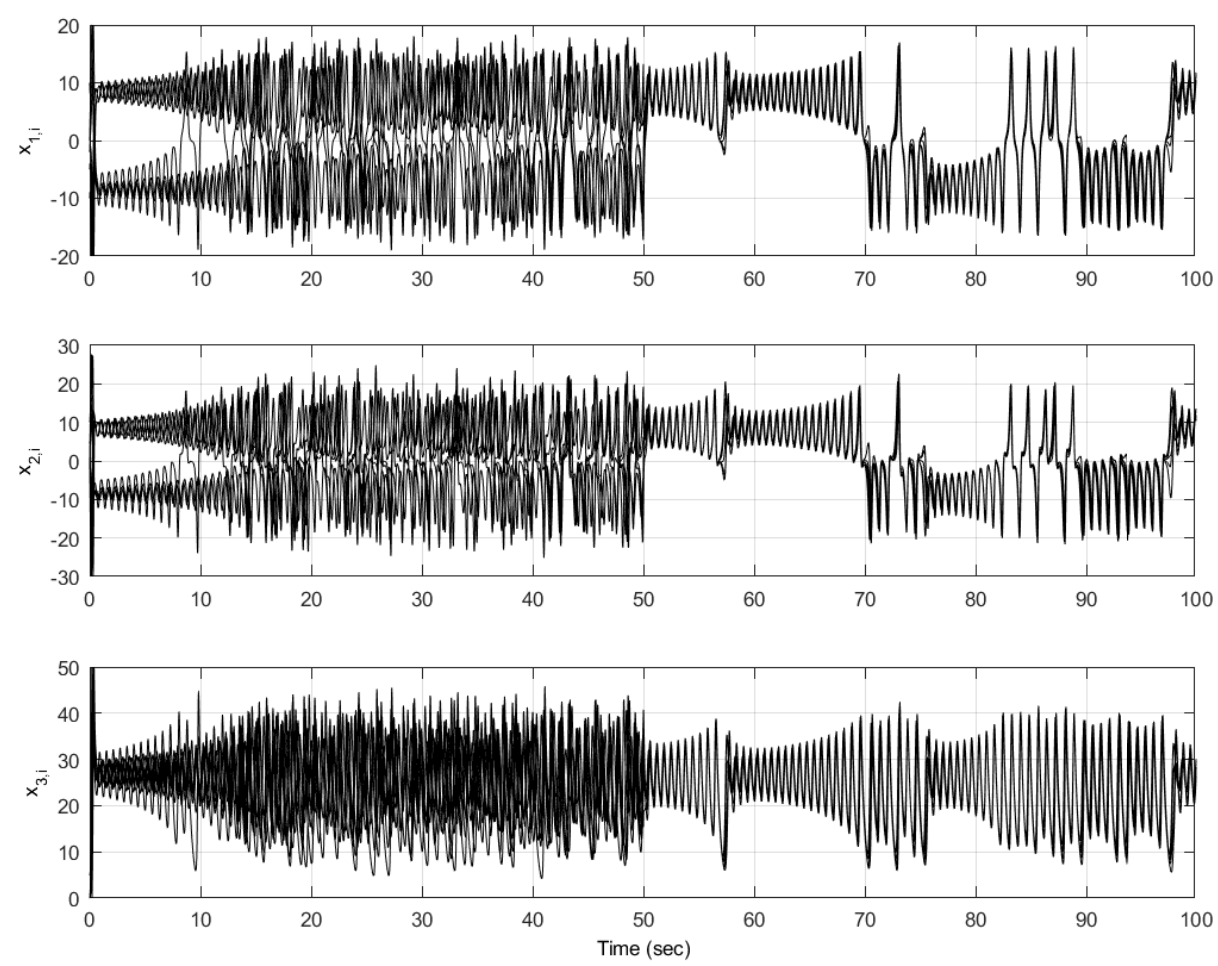

Figure 8. Synchronization state of the network for evolutionary couplings.

To verify the stability of the synchronization state, from the Proposition 1 , the parameter $\bar{d}$ is obtained in order to reach exponential stability of the synchronization regime. First the Jacobian matrix of system (7) at the virtual solution $s(t)$ is given by

$$
D f(s(t))=\left[\begin{array}{ccc}
-\sigma & \sigma & 0 \\
\rho-s_{3} & -1 & -s_{1} \\
s_{2} & s_{1} & -\beta
\end{array}\right]
$$

Matrix $\mathbf{G}=\kappa I_{3 \times 3}$ with $\kappa>0$. Now from Equation (3) the inequality is given by

$$
\left[\begin{array}{ccc}
2 \kappa(d-\sigma) & \rho+\sigma-s_{3} & s_{2} \\
\rho+\sigma-s_{3} & 2 \kappa(d-1) & 0 \\
s_{2} & 0 & 2 \kappa(d-\beta)
\end{array}\right] \leq-\alpha I_{3} .
$$

Therefore, to verify the exponential stability of the synchronization state a value for parameter $\bar{d}$ satisfying $\lambda_{2} \leq \bar{d}<0$ is required. 
To determine the stability of the synchronization state, let us depart from the Sylvester's criterion over the matrix (15), from where if the leading principal minors satisfy $(-1)^{k} \Delta_{k}>0$ for $k=1,2,3$ then matrix (15) is negative definite. For this case $\Delta_{k}$ are given as follows

$$
\begin{aligned}
& \Delta_{1}=2 \kappa(d-\sigma)<0 \\
& \Delta_{2}=4 \kappa^{2}(d-\sigma)(d-1)-\left(\rho+\sigma-s_{3}\right)^{2}>0 \\
& \Delta_{3}=8 \kappa^{3}(d-\sigma)(d-1)(d-\beta)-2 s_{2}^{2} \kappa(d-1)-2 \kappa\left(\rho+\sigma-s_{3}\right)^{2}(d-\beta)<0,
\end{aligned}
$$

for these inequalities to hold, note that $d \leq \bar{d}<0, s_{3}>0$ and considering $\kappa=\sigma$ and $d=-\beta$ satisfies the criterion and the matrix is negative definite, since $\lambda_{2} \leq d<0$, and for this example $\lambda_{2}(t)$ is illustrated in Figure 9; note that the eigenvalue does not satisfies the criterion for exponential stability for all time, however the synchronization is still stable. This behavior in the eigenvalue is due to the coupling function, since the coupling evolves accordingly to the behavior of the connected nodes, note that when the coupling in Figure 7 are close to zero, the value for $\lambda_{2}$ is also close to zero which means that the nodes are weakly connected and then the synchronization loses stability. This behavior on the stability of the synchronization is consequence of the evolutionary character of the couplings since these are not influenced or imposed to the network.

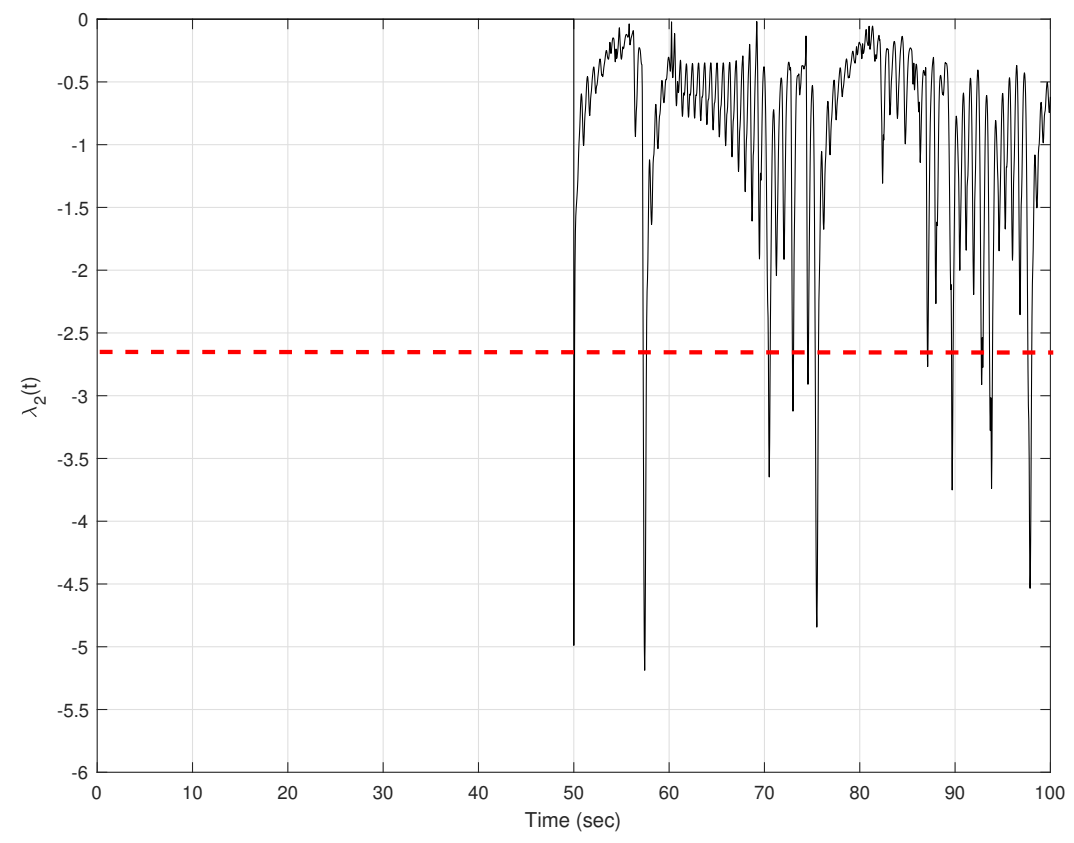

Figure 9. Time evolution of the eigenvalue $\lambda_{2}(t)$, dashed line divides the exponential stability region for $\lambda_{2}(t)$ greater than $d$ and stability for $\lambda_{2}(t)$ lees than $d$.

It is important to express that this result on the value of the parameter $d$ also applies to the case of time variation couplings (chaotic couplings), since the analysis is over the Jacobian matrix and are independent to the connectivity matrix, therefore, for that case, the exponential stability is completely achieved, this is $\lambda_{2}(t)<d=-\beta=-8 / 3 \approx-2.6$ for all $t>0$.

\section{Discussion}

The synchronization between nodes is achieved in both cases, one for imposed or external (exogenous), and the other defined by the connected nodes (endogenous). However, there is an important issue to be discussed, even if the synchronization is achieved, for the case of non autonomous exogenous couplings, the synchronization state is exponentially stable, in Figure 6 the behavior of the second larges eigenvalue is illustrated and the condition $\lambda_{2}(t)<d$ is satisfied for all time. On the other 
hand, the synchronization with evolutionary couplings is achieved, however, the synchronization is not exponentially stable for all time, this is the condition $\lambda_{2}(t)<d$ is not satisfied for all $t>0$ as it is shown in Figure 9, where the red line dives the exponential stability from the stability of the synchronization state, this is due to the parameters which define the value $d$. The synchronization is not lost since $\lambda_{2}(t)<0$ and there are not isolated nodes.

Note that for non autonomous exogenous couplings, the synchronization is controlled by the selection of the couplings, in other words it is always possible to fix the value of the couplings in order to satisfy the condition $\lambda_{2}(t)<d$ for all $t>0$ whereas in case of evolutionary couplings, the synchronization is reached by the collectivity of the nodes and as the couplings are not controlled or imposed the synchronization can or cannot be exponentially. These scenarios are directly consequence of the couplings properties.

It is important to mention that the synchronization is achieved in presence of non autonomous couplings or couplings depending on the behavior of the network, the stability of the synchronization manifold is achieved depending on the magnitude of the coupling strength between nodes, however, the meaning is different, on the one hand, non autonomous couplings describes an imposition of the behavior of the coupling strength, this is, that the nodes in the network obey the strength and the nodes do not have any possibility of changing their coupling, on the other hand, when the couplings depend on their own dynamics they define the couplings strength along time, this is called the evolution of the coupling and it is an intrinsic property without any external influence. An example for the non autonomous coupling case which also can be seen as an imposition of the strength of the couplings in a network is found in treatments where deep brain stimulation is performed to treat Alzheimer's, Parkinson's disease, tremor, and so forth, this stimulation is imposed or dictated to the network [18-20]. On the other hand, evolving couplings are considered when the nodes define their own coupling strength according to their requirements, this is, the relationships between agents are determined by them, for instance in social networks [21], in problems of formation control of UAV [26] and so forth.

\section{Conclusions}

In this contribution, we present the synchronization in complex networks with non autonomous and evolutionary (autonomous) couplings. These two ways to connect nodes in a network allow us to model natural phenomena more realistically. On one hand, the non autonomous couplings are considered unpredictable functions along time defined exclusively by an exogenous dynamics in this case a chaotic system. In this sense, the behavior of the nodes and the collectivity behavior do not define or influence the coupling values. Therefore, the connections between nodes are dictated by an external system; moreover, the resulting system that model the entire network is nonlinear time-varying however, the stability of the synchronization is completely determined by such a couplings values. On the other hand, for the case of pure evolution, the couplings were defined by the connected nodes, this is, the nodes involved define their strength coupling. The nodes define the coupling as they require, no more no less. This can be seen as a dynamical behavior where the coupling is strong or weak when the nodes require it, to achieve synchronization, but the exponential stability of the synchronization is not guaranteed, then it can be considered as a natural behavior of an evolutionary couplings. In this work, we present two models for the couplings between nodes of a network of dynamical systems: a model for a network with non autonomous couplings and a model for an evolutionary couplings. It was shown that the synchronization phenomenon in these networks is possible. The difference between non autonomous and evolution of the couplings was described in terms of the second largest eigenvalue and the importance of classifying the way of connecting nodes was stressed mainly due to the stability point of view. 
Author Contributions: Conceptualization and methodology, G.S.-P. and J.L.Z.; software, validation, J.L.Z.; formal analysis, G.S.-P. and G.O.-P.; investigation, G.S.-P.; writing-original draft preparation, G.S.-P. All authors have read and agreed to the published version of the manuscript.

Funding: This research received no external funding.

Conflicts of Interest: The authors declare no conflict of interest.

\section{References}

1. Wiener, N. Cybernetics:or Control and Communication in the Animal and the Machine, 2nd ed.; The MIT Press: Cambridge, MA, USA, 1995.

2. Winfree, A.T. Biological rhythms and the behavior of populations of coupled oscillators. J. Theor. Biol. 1967, $16,15-42$.

3. Travers, J.; Milgram, S. An experimental study of the small world problem. Sociometry 1969, 32, 425-443.

4. Strogatz, S. Sync: The Emerging Science of Spontaneous Order; Hyperion: New York, NY, USA, 2003.

5. Strogatz, S.; Garcia-Ojalvo, J.; Elowitz, M.B. Modeling a synthetic multicellular clock: Repressilators coupled by quorum sensing. Proc. Natl. Acad. Sci. USA 2004, 1, 10955-10960.

6. Díaz-Guilera, A.; Arenas, A. Phase patterns of coupled oscillators with application to wireless communication. In Bio-Inspired Computing and Communication; Springer: Berlin Heidelberg, Germany, 2008; pp. 184-191.

7. Watts, D.J.; Strogatz, S.H. Collective dynamics of small-world networks. Nature 1998, 393, 440—442. [PubMed]

8. Barabási, A.L.; Albert R. Emergence of scaling in random networks. Science 1999, 286, 509-512.

9. Wang, X.F.; Guanrong, C. Synchronization in scale-free dynamical networks: Robustness and fragility. IEEE Trans. Circ. Syst. I Fundam. Theory Appl. 2002, 49, 54-62.

10. Wang, X.F.; Guanrong, C. Synchronization in small-world dynamical networks. Int. J. Bifurc. Chaos 2002, 12, 187-192.

11. Osipov, G.V.; Kurths, J.; Zhou, C. Synchronization in Oscillatory Networks; Springer Science \& Business Media: Berlin/Heidelberg, Germany, 2007.

12. Lu, J.; Chen, G. A time-varying complex dynamical network model and its controlled synchronization criteria, IEEE Trans. Autom. Control 2005, 50, 841-846.

13. Anzo, A.; Barajas-Ramirez, J.G. Synchronization in complex networks under structural evolution. J. Frankl. Inst. 2014, 351, 358-372.

14. Dorogovtse, S.N.; Mendes, J.F. Evolution of Networks: From Biological Nets to the Internet and WWW; Oxford University Press: New York, NY, USA,2013.

15. Sorrentino, F.; Ott, E. Adaptive synchronization of dynamics on evolving complex networks. Phys. Rev. Lett. 2008, 100, 114101.

16. Jiménez-Martín, M.; Rodríguez-Laguna, J.; D’Huys, O.; de la Rubia, J.; Korutcheva, E. Synchronization of fluctuating delay-coupled chaotic networks. Phys. Rev. E 2020, 95, 052210.

17. Gan, L.; Li, S.; Duan, N.; Kong, X. Adaptive Output Synchronization of General Complex Dynamical Network with Time-Varying Delays. Mathematics 2020, 8, 311.

18. Scharrea, D.W.; Weichartb, E.; Nielsonc, D.; Zhangd, J.; Agrawale, P.; Sederbergb, P.B.; Knoppd, M.V.; Rezaic, A.R. Deep Brain Stimulation of Frontal Lobe Networks to Treat Alzheimer's Disease. J. Alzheimers Dis. 2018, 62, 621-633.

19. Follett, K.A.; Weaver, F.M.; Stern, M.; Hur, K.; Harris, C.L.; Luo, P.; Marks, W.J., Jr.; Rothlind, J.; Sagher, O.; Moy, C.; et al. Pallidal versus Subthalamic Deep-Brain Stimulation for Parkinson's Disease. N. Engl. J. Med. 2010, 362, 2077-2091. [PubMed]

20. Dougherty, D.D.; Rezai, A.R.; Carpenter, L.L.; Howland, R.H.; Bhati, M.T.; O’Reardon, J.P.; Eskandar, E.N.; Baltuch, G.H.; Machado, A.D.; Kondziolka, D.; et al. A Randomized Sham-Controlled Trial of Deep Brain Stimulation of the Ventral Capsule/Ventral Striatum for Chronic Treatment-Resistant Depression. Biol. Psychiatry 2015, 78, 240-248.

21. Arias, N.; Calvo, M.D.; Benítez-Andrades, J.A.; Álvarez, M.J.; Alonso-Cortés, B.; Benavides, C. Socioeconomic Status in Adolescents: A Study of Its Relationship with Overweight and Obesity and Influence on Social Network Configuration. Int. J. Environ. Res. Public Health 2018, 15, 2014.

22. Belykh, I.; Di Bernardo, M.; Kurths, J.; Porfiri, M. Evolving dynamical networks. Physica D 2014, 267, 1-6. 
23. Lehnertz, K.; Ansmann, G.; Bialonski, S.; Dickten, H.; Geier, C.; Porz, S. Evolving networks in the human epileptic brain. Physica D 2014, 267, 7-15.

24. Skardal, P.S.; Taylor, D.; Restrepo, J.G. Complex macroscopic behavior in systems of phase oscillators with adaptive coupling. Physica D 2014, 267, 27-35.

25. FanWang, X.; Chen, G. Complex networks: Small-world, scale-free and beyond. IEEE Circ. Syst. Mag. 2003, 3, 6-20.

26. Park, S.; Kim, K.; Kim, H.; Kim, H. Formation Control Algorithm of Multi-UAV-Based Network Infrastructure. Appl. Sci. 2018, 8, 1740. [CrossRef]

Publisher's Note: MDPI stays neutral with regard to jurisdictional claims in published maps and institutional affiliations.

(C) 2020 by the authors. Licensee MDPI, Basel, Switzerland. This article is an open access article distributed under the terms and conditions of the Creative Commons Attribution (CC BY) license (http:/ / creativecommons.org/licenses/by/4.0/). 